\title{
Methods of fomalization and simulation of telecommunication network using network slice
}

\author{
Anastasiia Zhuravel \\ Institute of Telecommunication Systems \\ Igor Sikorsky Kyiv Politechnic Institute \\ Kyiv, Ukraine \\ anseezhur@gmail.com
}

\author{
Mariia Skulysh \\ Institute of Telecommunication Systems \\ Igor Sikorsky Kyiv Politechnic Institute \\ Kyiv, Ukraine \\ mskulysh@gmail.com
}

\begin{abstract}
The 5G networks are already widely used and developed for practical use and implementation in modern communication systems. One of the key technologies of these networks is network slicing technology that allows you to distribute, optimize and improve the operation of the entire network. There are many representations and implementations of network slicing. In this paper, we consider models and methods of slicing that can be used for implementation in telecommunication networks.
\end{abstract}

Each of the considered models has both advantages and limitations. Further studies can be devoted to possible modifications of these models from a client-oriented point of view and to considering the possibility of combining these models depending on the requirements of the network.

Keywords—-network slicing, slice, $5 G$.

\section{INTRODUCTION (HEADING I)}

Nowadays, the number of Internet users are rapidly increasing along with the number of devices we use to get access to the Internet. The growing number of network services, more strict requirements to the quality of service and to the speed of the fixed and mobile Internet-access have caused the modernization of the network architecture, based on new requirements, standards and recommendations. Due to the active development of the Internet of Things concept, devices connected to the Internet generate huge volumes of network traffic, which makes the further usage of the networks built only on routers and switches impossible.

It becomes one of the most important reasons of active development of the Software Defined Networks (SDN) and Network Functions Virtualization Services (NFVS), which are integral elements of the fifth generation network concept [1]

With these technologies, 5G networks can provide the necessary programmability, flexibility, scalability, etc., that are needed to build different logical (virtual) networks for a specific type of task, without changing the infrastructure platform. These logical networks with shared resources are network slices. The purpose of this work is to analyze the current models and methods of network slicing in modern telecommunication networks, and their implementations.

\section{NETWORK SLICING}

The slicing concept in communications networks was previously proposed in contexts of distributed services and applications architectures; but its usage in mobile networks is the newest. From the mobile operator point of view, slicing is the creation of a user-friendly needs, a set of logically independent networks (network slices) placed along a common physical infrastructure; each of which is configured to represent certain services. The concept of network slicing is shown in fig. 1 [2].

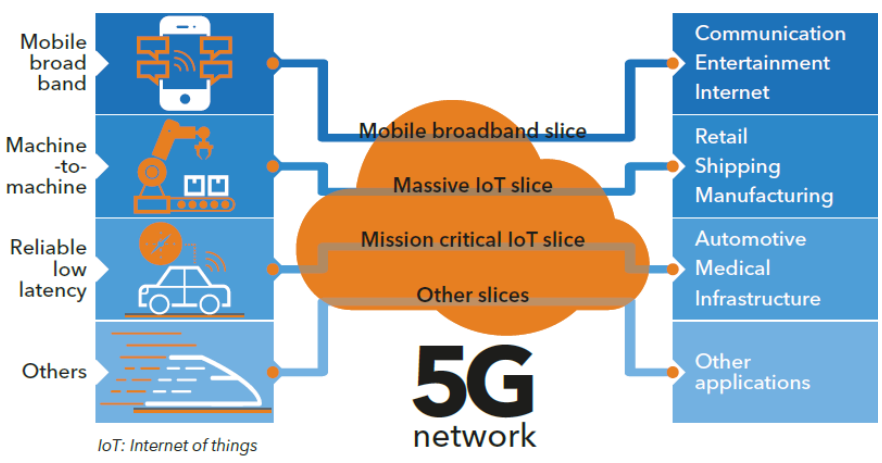

Fig 1. Network slicing concept

III. SDN AND NFV INTEGRATION INTO SLICING TECHNOLOGY IN COMMUNICATION NETWORKS

Software-Defined networking (SDN) and Network Function Virtualization are considered to be the most perspective technologies in the modern and future telecommunication networks, where virtualized infrastructures and services provide unprecedented network flexibility and programmability.

Over the past few years, SDN and NFV have been improved by unique interaction of standardization organizations with software developers communities, who is changing the methods of implementation of new technologies together. 
Innovative industry groups such as the ETSI ISG group (Industry Specification Group) for the NFV and ONF (the Open Networking Foundation) organization for the SDN created reference architectures, substantiated usage scenarios and changed the requirements for the components that are an integral part of NFV and SDN. The SDN network architecture supports the principles of network slicing because SDN allows you to manage a common infrastructure network and effectively support multiple client instances of the network [8].

Fig. 2. illustrates an example of integrating SDN and NFV technologies for implementing slicing in $5 \mathrm{G}$ communication networks [9].

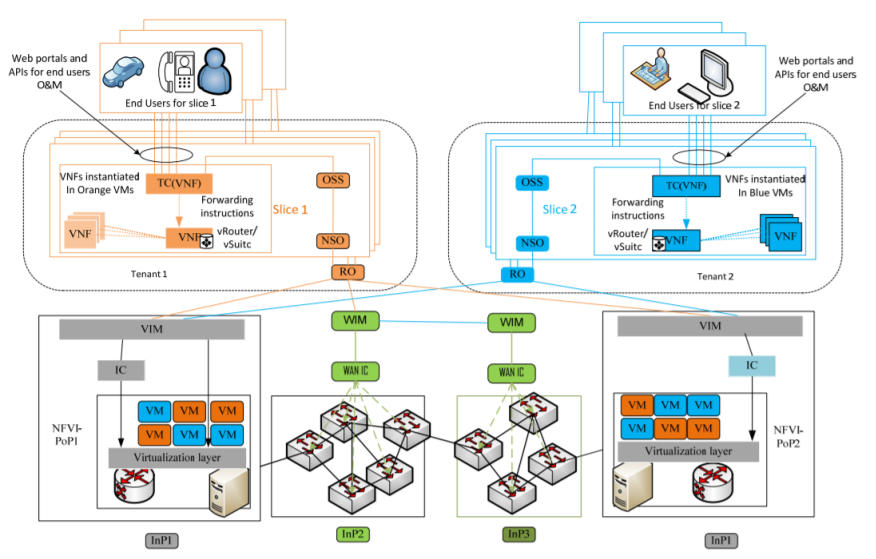

Fig 2. Network slice architecture for SDN and NFV

\section{Network SLiCING MEthods AND ModelS}

\section{A. Uniform Reference Model}

Define Describes all of the functional elements and instances of a network slice in NFV. Describes shared nonsliced network parts [3].

In this model a network slice is considered as a combination of subsets of network resources and virtual network functions. Network slicing in this case is the creation and management of such slices on top of the physical network infrastructure (Fig. 1).

Mathematically, a slice of a single reference model is described as follows:

$$
s=<N R, N F, R>\text {, }
$$

where $\mathrm{s}$ is a network slice, NR is a set of network resources, $\mathrm{NF}$ is a set of network functions, $\mathrm{R}$ is a set of relations linking network resources and network functions to each other.

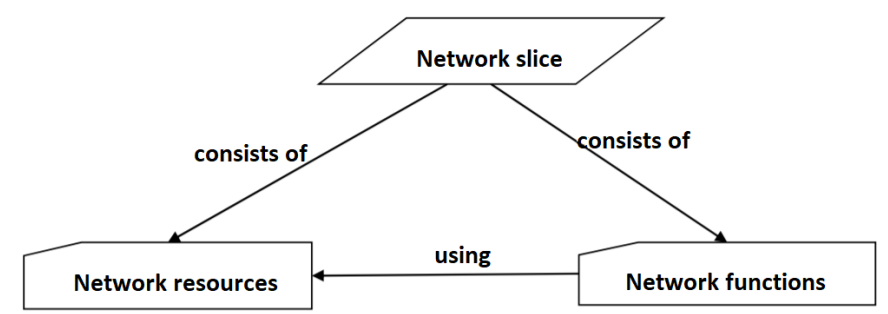

Fig 3. Uniform Reference Model of a network slice [6].

\section{B. Modified Reference Model}

This model extends the reference model, in this model slice is considered as a combination of subsets of network resources, virtual network functions and network services for the given time. Network slicing in this case is the process of creating and managing these extended slices on top of the physical network infrastructure.

Mathematical model:

$$
s=<M F, N R, N F, N S, R>\text {, }
$$

where $\mathrm{s}$ is a network slice, MF is a set of management functions, NR is a set of network resources, NF is a set of network functions, NS is a set of network services, $\mathrm{R}$ is a set of relations linking network resources and network functions, as well as network resources and network services [4].

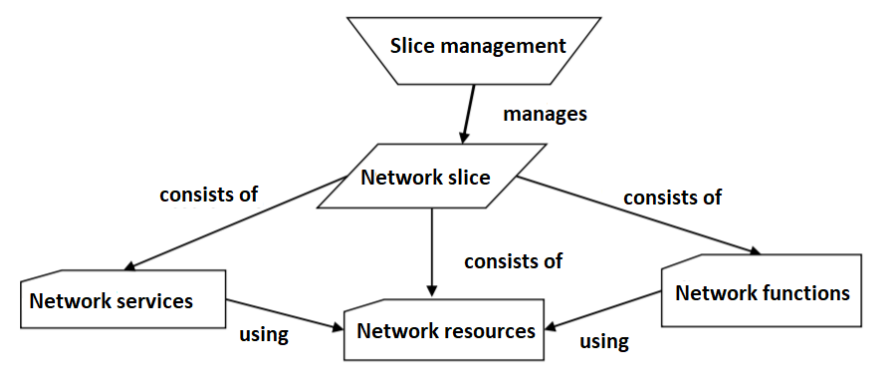

Fig 4. Modified Reference Model of a network slice [6].

\section{Slice model with one S/D pair}

The coexistence of several network slices on the basis of one physical network is considered in this model. So, each slice provides network traffic between only one source (Source) and only one receiver (Destination), that is, the slice is represented by a source/destination (S / D) pair. The definition of each slice includes a unidirectional linked list of virtual network functions (VNFs), which determines the sequence of execution of different VNFs.

Mathematical model:

$$
s=\langle S, D, N R, N F C, R>\text {, }
$$


where $\mathrm{S}$ is a network slice, $\mathrm{S}$ is a traffic source, $\mathrm{D}$ is a traffic receiver, NR is a set of network resources, NFC is a sequence (linked list) of network functions, $\mathrm{R}$ is a set of relations connecting the elements of the model with each other $[5]$.

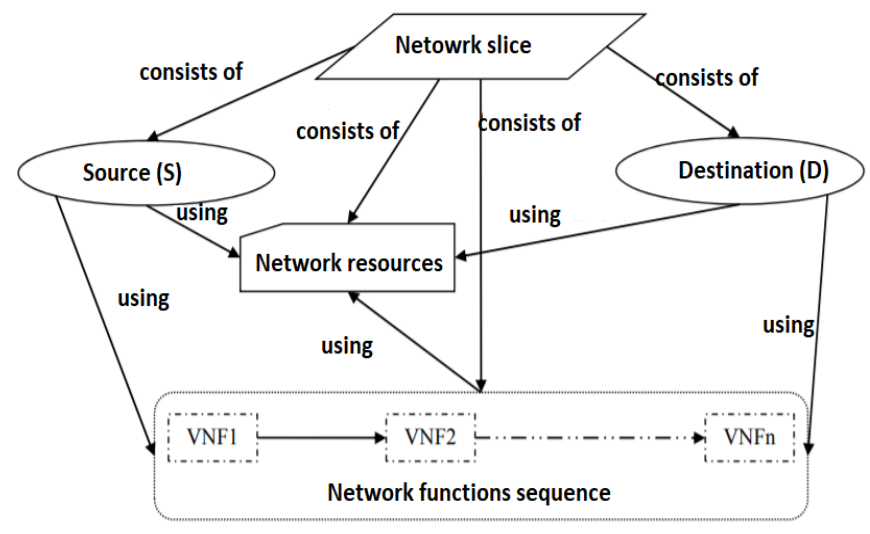

Fig 5. Slice model with one S/D pair [6].

\section{Slice model with a few $S / D$ pairs}

In this model, each slice provides network traffic between multiple sources (Sources) and multiple receivers (Destinations), i.e. the slice is represented by a set of sourcedestination (S / D) par. This model is a development of the previous one. Each slice of a model with many S / D pairs consists of slices of the previous type, i.e. the type of one S / D pair. Network slicing in this case is the creation and management of such slices on top of the physical network infrastructure [6].

Mathematical model:

\section{$\mathrm{S}=\{\mathrm{x} \mid \mathrm{x}$ this is a slice of type of one $\mathrm{S} / \mathrm{D}$ pair $\}$,}

where $\mathrm{s}$ is a network slice.

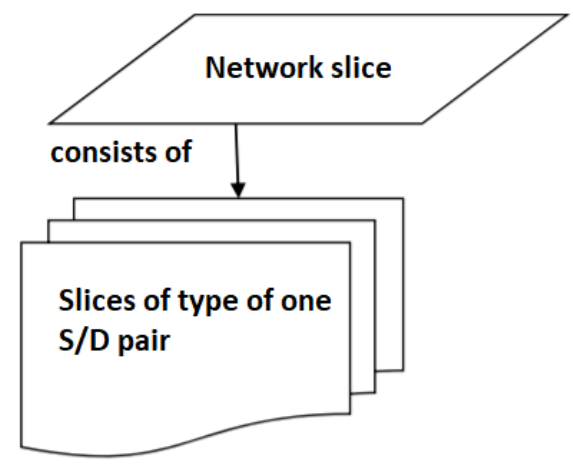

Fig 6. Slice model with a few S/D pairs [6].

\section{E. Content-oriented Model}

In content-oriented (or information-oriented) network slicing, network resources are allocated to content. So there are content-oriented resources, which include caching resources and communication resources, which are used to transfer content. In this model, each network slice is associated with only one specific content and consists of two internal sub-sectors: a slice of caching and a slice of communication. Network slicing in this case is the process of creating and managing information-oriented slices for each content on top of the physical network infrastructure [7].

Mathematically content-oriented slice is described by the following tuple:

$s=\langle s 1, s 2, N R, C O C N R, C O C o m N R, C O C N F, C O C o m N F, R\rangle$

where $\mathrm{s}$ is a content-oriented network slice, s1 (internal slice of a network slice) - content-oriented caching slice, s2 (internal slice of a network slice) - content-oriented communication slice, NR - the set of all network resources, $\mathrm{COCNR} \subset \mathrm{NR}$ is a set of content-oriented caching resources, COComNR $\subset \mathrm{NR}$ is a set of content-oriented communication resources, COCNF is a set of content-oriented caching functions, COComNF is a set of content-oriented communication functions, $\mathrm{R}$ is a set of relations linking content-oriented network resources and content-oriented network functions, as well as internal slices and contentoriented network resources and functions.

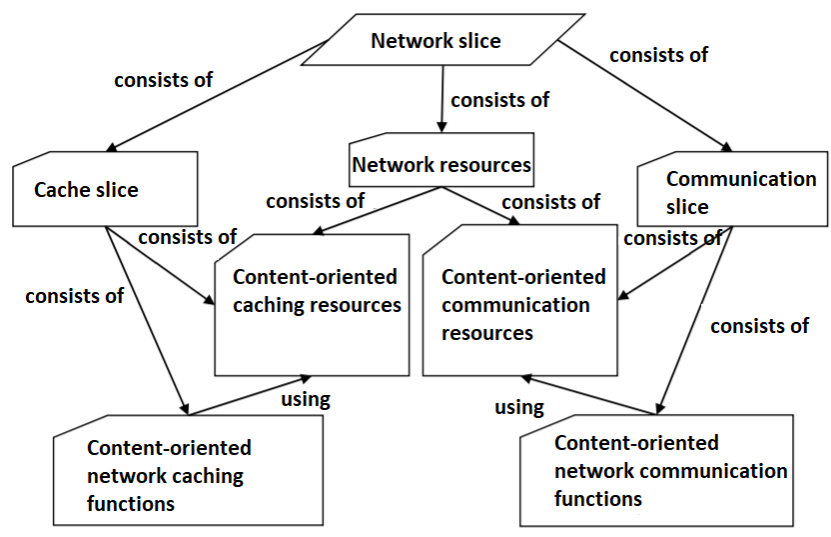

Fig 7. Content-oriented Model of a network slice [6].

\section{CONCLUSIONS}

In this work, modern models of network slicing were considered, all reviewed models are relevant from the practical perspective and physical implementation in modern telecommunication systems. All models have their pros and cons, and depending on this can be used for various purposes and tasks.

The Uniform Reference Model is easy to learn and implement, has high flexibility and scalability, but does not 
allow achieving the maximum optimization, since the network services layer is not clearly presented and there are no clear methods for optimizing most of the parameters and separating control and traffic. Partially, these issues are resolved in the Modified Reference Model, with the exception of parameters optimization methods.

Concerning the model of one S / D pair, it is just as easy to implement as the two previous models, but it is resistant to the uncertainties of traffic requirements and has a mathematical and semantic representations, it is possible to add into the model different relations between components for slices control and monitoring. However, at the same time, the model has a limitation in the number of services and a rigidly defined sequence of execution of virtual functions.

In the model of many S / D pairs, the limitations of the previous model are retained in addition to the fact that many $\mathrm{S} /$ D pairs can be associated to one slice, there is a good traffic isolation and an auto slice scaling algorithm.

As for the content-oriented model, it has limitations in the ability to provide only one service and requires additional resources for caching. At the same time, the model has high reliability, speed, mathematical and semantic representations, and the possibility to add into the model different relations between components for slices control and monitoring.

This paper gives us an overview of modern slice models with a clearer understanding of their limitations and advantages. In subsequent studies, the implementation and merging of various models for different services in a single network can be considered. As well as consideration of possible modifications of the basic models to bypass their limitations and meet the requirements and users needs. Such models as: nominal, $\Gamma$-robust approach, easy reliable models are considered to be unsuitable for practical implementations and are not considered in this work.

\section{REFERENCES}

[1] L. Globa, S. Sulima, M. Skulysh, A. Zhuravel, «An Approach for Virtualized Network Slices Planning in Multiservide Communication Environment», Information and Telecommunication Sciences, pp. 37 44, 2019.

[2] P. Ashwood-Smith, "Why end-to-end network slicing will be important for 5G”, ITUNews, 2017.

[3] A.Galis, "Key Challenges - Reliability, Availability and Serviceability in Network Slicing”, NetSoft2017, 2017.

[4] Kotulski, Z., "Towards Constructive Approach to End-to-End Slice Isolation in 5G Networks," Journal on Information Security. 2018.

[5] M. Leconte, G. Paschos, P. Mertikopoulos, U. Kozat, "A Resource Allocation Framework for Network Slicing", USA.

[6] S. Muhizi1, R. Kirichek, "Comparative Evaluation of Models and Methods of Network Slicing”, 2018

[7] Jin, H., Lu, H, Zhao, "Content-Oriented Network Slicing Optimization based on CacheEnabled Hybrid Radio Access Network" EURASIP Journal on Wireless Communications and Networking. 2018.

[8] S. Muhizi, R. Kirichek, "Analysis of Network Slicing Technology for 5G Networks", 2017.

[9] Vladyko A., Muthanna A., Kirichek R. Comprehensive SDN Testing Based on Model Network // Lecture Notes in Computer Science. 2016. 